Research Paper

\title{
The influence of individual socioeconomic status on the clinical outcomes in ischemic stroke patients with different neighborhood status in Shanghai, China
}

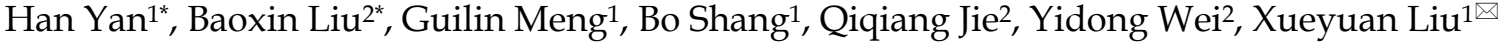 \\ 1. Department of Neurology, Shanghai Tenth People's Hospital, Tongji University School of Medicine, Shanghai, 200072, China \\ 2. Department of Cardiology, Shanghai Tenth People's Hospital, Tongji University School of Medicine, Shanghai, 200072, China \\ * Both Han Yan and Baoxin Liu contributed equally to this work and should be considered co-first authors. \\ $\triangle$ Corresponding author: Professor Xueyuan Liu, MD, PhD. Email: liuxy@tongii.edu.cn. Address: Department of Neurology, Shanghai Tenth People's Hospital, \\ Tongji University School of Medicine, 301 Middle Yanchang Road, Shanghai, 200072, China. Tel: +86-21-66306920; Fax: +86-21-66307239 \\ (c) Ivyspring International Publisher. This is an open access article distributed under the terms of the Creative Commons Attribution (CC BY-NC) license \\ (https://creativecommons.org/licenses/by-nc/4.0/). See http://ivyspring.com/terms for full terms and conditions.
}

Received: 2016.08.17; Accepted: 2016.11.24; Published: 2017.01.15

\begin{abstract}
Objective: Socioeconomic status (SES) is being recognized as an important factor in both social and medical problems. The aim of present study is to examine the relationship between SES and ischemic stroke and investigate whether SES is a predictor of clinical outcomes among patients with different neighborhood status from Shanghai, China.

Methods: A total of 471 first-ever ischemic stroke patients aged 18-80 years were enrolled in this retrospective study. The personal SES of each patient was evaluated using a summed score derived from his or her educational level, household income, occupation, and medical reimbursement rate. Clinical adverse events and all-cause mortality were analyzed to determine whether SES was a prognostic factor, its prognostic impact was then assessed based on different neighborhood status using multivariable Cox proportional hazard models after adjusting for other covariates.

Results: The individual SES showed a significant positive correlation with neighborhood status $(r=$ 0.370; $P<0.001$ ). The incidence of clinical adverse events and mortality were significantly higher in low SES patients compared with middle and high SES patients $(P=0.001$ and $P=0.037$, respectively). After adjusting other risk factors and neighborhood status, Kaplan-Meier analysis showed clinical adverse events and deaths were still higher in the low SES patients (all $P<0.05$ ). Multivariate Cox regression analysis demonstrated that both personal SES and neighborhood status are independent prognostic factors for ischemic stroke (all $P<0.05$ ). Besides, among patients with low and middle neighborhood status, lower individual SES was significantly associated with clinical adverse events and mortality (all P < 0.05).

Conclusion: Both individual SES and neighborhood status are significantly associated with the prognosis after ischemic stroke. A lower personal SES as well as poorer neighborhood status may significantly increase risk for adverse clinical outcomes among ischemic stroke patients.
\end{abstract}

Key words: Ischemic stroke; Socioeconomic status; Neighborhood status; China; Health inequality; Survival.

\section{Introduction}

Stroke has been recognized as one of the major causes of morbidity and mortality in the world. In China, the burden of stroke is particularly serious and the mortality is higher when compared with the world average [1]. However, declining stroke incidence is rarely observed, which is in part due to the rapidly aging population. Thus, there is an increase in the number of stroke survivors who require long-term, costly care. Although there exist differences among three subtypes of stroke (ischemic stroke, intracerebral hemorrhage, and subarachnoid hemorrhage), ischemic stroke has been reported to be 
with the highest incidence and represent most of all stroke events due to vascular thrombosis and occlusion in brain [2-4]. Despite advances in evidence-based pharmacological and interventional therapies, ischemic stroke patients still suffer from a high risk of hospitalization and reduced quality of life.

Since ischemic stroke patients are at high risk of recurrent incidence and neuropsychiatric complications, it is important to comprehensively evaluate the risk factors. The controllable factors are consisted of hypertension, dyslipidemia, diabetes mellitus, atrial fibrillation, smoking habit, obesity, lack of physical exercise. Other uncontrollable factors such as age, gender, family history, psychosocial factors have also been recognized. Apart from these demographic, physiological and psychological factors, an individual's socioeconomic status (SES) is also associated with his or her lifestyle and health behavior that could lead to stroke and affect clinical outcomes. SES refers to a personal social position relative to other members of a society, which is generally determined by education, income, occupation and social status [5]. Accumulating evidence demonstrated that lower SES is associated with vascular risk factors and comorbidities that contribute to higher stroke incidence and are likely to decrease the survival rate by $30 \%$ after stroke [6-9]. More recently, several studies have suggested a closely association between lower SES and worse functional impairment after stroke [4, 10, 11]. In addition, low educational level and occupational status are interrelated with household income and may have a synergistic effect on health [12].

Over recent decades, socioeconomic factors have aroused interest in the field of healthcare as the health inequalities were increasing in China [13-15]. Among these inequalities, the rural-urban health inequality is prominent and people from rural areas were often considered low SES due to low educational level, work status, household income, and medical insurance reimbursement [16, 17]. In fact, people from different areas have diverse neighborhood status and possess disparate neighborhood-based resources including education, employment, housing, and medical care that closely associated with personal SES [18]. Stafford et al. [19] have examined the association between socioeconomic characteristics and personal health status by taking into consideration of both neighborhood status and individual SES. The results showed neighborhood status also impacts individual SES and the residents with a higher individual SES from affluent neighborhoods would indicate much better health status.

$$
\text { Although a neighborhood is generally }
$$

considered as a geographically localized community that residents lived in, however, there is a tendency to describe a Chinese patient's neighborhood status using the China's household system, or hukou system regardless of where he or she currently lived, since the healthcare-related strategies such as health insurance reimbursement mainly depended on the policies issued in hukou registered locations [20]. Despite huge number of rural-to-urban migrants are living in large cities of China such as Peking, Shanghai and Guangzhou, they are still carrying their original rural hukou locations. Their neighborhood status that influencing healthcare are actually associated with these original hukou registered locations rather than the current residence [19]. Thus, it is more reasonable to describe the neighborhood status using an individual's hukou status in these cities. In the meantime, this complexity in neighborhood status could have possibly altered the personal SES of ischemic stroke patients, and thus the clinical outcomes may be hugely influenced. However, most previous studies centered on the relationship between SES and ischemic stroke were mainly conducted in high-income and developed countries and the indicators used in these studies may not be applied in such conditions in China. Besides, several findings from the existing studies have also been inconsistent $[9,11,21,22]$. In the present study, we investigated the association between SES and clinical outcomes in ischemic stroke among patients with different neighborhood status from Shanghai, China.

\section{Methods and materials}

\section{Data source and patient population}

From September 2012 to August 2015, a total of 471 first-ever ischemic stroke patients aged from 18 to 80 years were enrolled and followed up in this retrospective study. All the participants had been hospitalized in the Department of Neurology, Shanghai Tenth People's Hospital, Tongii University School of Medicine. Patients documentation used for evaluation including demographic characteristics, cardiovascular risk factors, socioeconomic factors, admission history, physical examinations, treatment records, neurology consultations, and computed tomography/magnetic resonance imaging (CT/MRI) reports were collected. Ischemic stroke was defined according to 2013 American Heart Association/ American Stroke Association Guidelines and 2013 Updated Definition [23, 24], which described ischemic stroke as an acute onset and rapidly developing clinical features of disturbances in neurologic functions lasting more than 24 hours and was confirmed as being to a cerebrovascular cause by 
CT/MRI. We excluded intracerebral hemorrhage and subarachnoid hemorrhage confirmed by brain CT/MRI. Transient ischemic attacks, silent brain infarction, and nonvascular diseases such as head trauma, blood disease, brain tumor, and seizures which could also lead neurological deficits, were also not included in present study. Patients with severe hepatic or renal failure were still not eligible in our study. The study was approved by the institutional ethics committee of Shanghai Tenth People's Hospital. Written informed consent was obtained from all patients.

\section{Clinical outcomes}

The primary outcomes were clinical adverse events including 1) death, 2) lone post-stroke disability, 3) lone recurrent nonfatal stroke, and 4) post-stroke disability + recurrent nonfatal stroke. The all-cause mortality was considered as the secondary endpoint. We followed the patients until January 1, 2016. Prescribed medication, clinical symptoms, and medical history were all gathered and necessary examinations were performed at each follow-up. Patients lost response during follow-up period were censored as alive on the last day of contact. The mRS was used as a global standard for measurements of disability which included six gradual grades in functional deficit of nervous system (0 refers to "no assistance needed", 5 refers to "constant care needed" and 6 refers to "death") [25]. We collected the results and identified mRS score based on the information provided by patients and reliable proxy relatives. A mRS score of 3-5 (assistance or constant care was required for basic daily living) was considered as post-stroke disability.

\section{Socioeconomic status measurements}

We gathered data on the following factors as indicators of individual SES: education, occupation, annual income, and medical insurance. Each factor was categorized to five groups from low to high level, for which a gradually increasing score (0-4) was assigned and the final summed score of each factor represented the individual SES. Level of education attainment: illiterate and semiliterate (low; score $=0$ ), primary school (medium-low; score $=1$ ), secondary school/specialized school (medium; score $=2$ ), high school/professional school (medium-high; score=3), and college/university or higher (high; score $=4$ ). Work status pre-stroke: peasants and unemployed (low; score $=0$ ); manual workers (medium-low; score $=1$ ); retired patients (medium; score $=2$ ); businessmen or clerks (medium-high; score $=3$ ); and managers, professionals, or government officers (high; score=4). Annual income: $<¥ 12,000$ (low; score $=0$; " $¥$ " refers to Renminbi, the official currency of China, which is equivalent to CNY, or Chinese Yuan); $¥ 12,000-¥ 36,000 \quad$ (medium-low; score $=1$ ); $¥ 36,000-¥ 60,000$ (medium; score $=2$ ); $¥ 60,000-¥ 120,000$ (medium-high; score $=3$ ); and $\geq ¥ 120,000$ (high; score=4). Medical insurance reimbursement rates: without medical insurance (low; score $=0$ ); $0-25 \%$ (medium-low; score $=1$ ); $25-50 \%$ (medium; score $=2$ ); $50-75 \%$ (medium-high; score $=3$ ); and $\geq 75 \%$ (high; score $=4$ ). We divided the study population into three groups according to the tertiles of score distribution (Figure 1): Low ( $\leq 7)$, Middle (8-9), and High groups $(\geq 10)$.

We furthermore analyzed and stratified the patients' neighborhood status into three groups according to the information on hukou registered locations: Low (village, town and rural areas); Middle (suburb and county areas); and High (district and urban areas). For the purposes of the present study, a participant's rural, suburb, or urban area was considered his or her neighborhood.

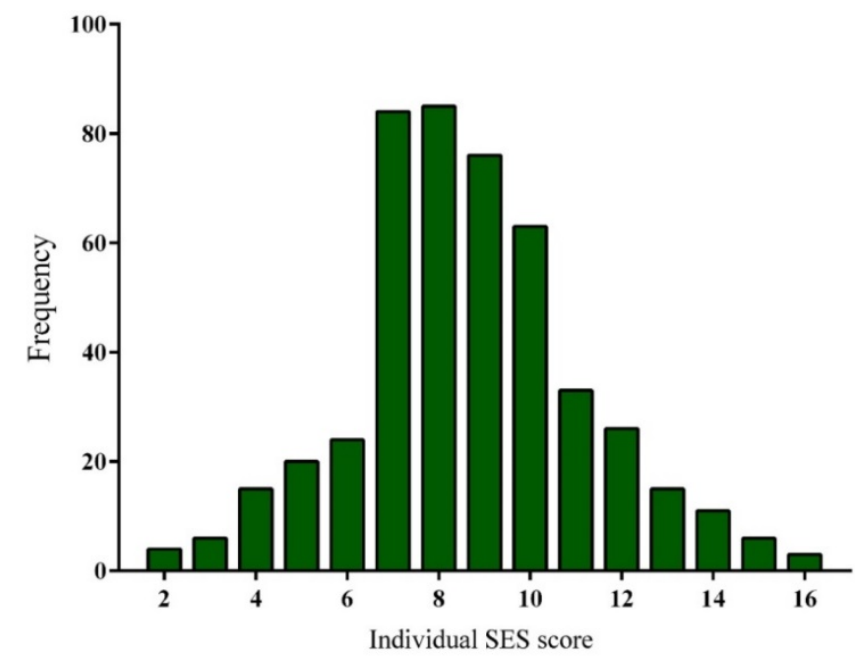

SES, socioeconomic status

Figure 1 - Distribution of individual SES scores among the enrolled 471 study population. SES, socioeconomic status.

\section{Definitions of cardiovascular risk factors}

Coronary heart disease (CHD) was diagnosed according to coronary angiography showed Luminal diameter narrowing $>50 \%$ in a major epicardial coronary artery due to stenosis, a history of confirmed myocardial infarction, or a history of revascularization by percutaneous coronary intervention or coronary artery bypass graft. Hypertension was diagnosed when blood pressure was $\geq 140 / 90 \mathrm{mmHg}$ or use of antihypertensive treatment. Diabetes mellitus was diagnosed according to a fasting plasma glucose $\geq 7.0 \mathrm{mmol} / \mathrm{L}$, or random 
plasma glucose $\geq 11.1 \mathrm{mmol} / \mathrm{L}$. Lipid disorders were defined as total cholesterol $\geq 5.7 \mathrm{mmol} / \mathrm{L}$, or LDL $\geq 3.6$ $\mathrm{mmol} / \mathrm{L}$, or $\mathrm{HDL}<1.04 \mathrm{mmol} / \mathrm{L}$, or patients were currently treated with anti-hyperlipidemic drugs. Tobacco use was defined by using $\geq 1$ pack of cigarettes per day at least 1 year.

\section{Statistical analysis}

In descriptive data analysis, we reported continuous variables as mean \pm standard deviation (SD) and categorical variables as a percentage. Differences across tertiles of individual SES were tested using one-way analysis of variance or a Kruskal-Wallis test for continuous variables and chi-square test for categorical variables. Event-free survival curves were constructed using the Kaplan-Meier method and assessed using the log-rank test. To determine the combined influences of individual SES and neighborhood status on clinical outcomes in ischemic stroke, multivariate Cox regression analysis was performed with the first step adjusted for age, gender and cardiovascular risk factors (Model 1), and then the second step adjusted for Model 1 plus individual SES and neighborhood status (Model 2). The 95\% confidence interval (CI) of the hazard ratio (HR) is reported for all of the significant risk factors. To assess the independent association between clinical outcomes and individual SES based on neighborhood status, we compare HR according to neighborhood status by individual SES using a multivariate Cox regression analysis adjusted for age, sex, cardiovascular risk factors, education, occupation, annual family income, and medical insurance reimbursement levels. To clarify an independent association between individual SES and clinical outcomes that excluded the socioeconomic influences of the neighborhood status, a multivariate Cox regression analysis adjusted for age, gender, and cardiovascular risk factors was also used to compare HR according to individual SES by neighborhood status. $\mathrm{P}<0.05$, which is two-sided, was considered significant. Statistical analyses were performed with the IBM SPSS version 20.0 (IBM Co., Armonk, NY, USA).

\section{Results}

\section{Baseline characteristics}

Among the 723 patients initially screened, 121 patients did not meet the requirements, 104 patients refused or expressed no interested, and 27 patients failed to provide essential data were excluded. The average age of finally enrolled 471 participants was $65.9 \pm 10.2$, and $51.6 \%$ were male. The demographic data, drug therapy and neighborhood status across the tertiles of individual SES were summarized in Table 1.

Table 1. Baseline characteristics according to the tertiles of individual socioeconomic status

\begin{tabular}{|c|c|c|c|c|}
\hline \multirow[b]{2}{*}{ Variables } & \multicolumn{4}{|c|}{$\begin{array}{l}\text { Individual socioeconomic status } \\
\text { tertiles }\end{array}$} \\
\hline & $\begin{array}{l}\text { Low } \\
(n=153)\end{array}$ & $\begin{array}{l}\text { Middle } \\
(\mathrm{n}=161)\end{array}$ & $\begin{array}{l}\text { High } \\
\text { (n=157) }\end{array}$ & $\begin{array}{l}P \\
\text { value }\end{array}$ \\
\hline Age, yrs (mean \pm SD) & $66.7 \pm 10.1$ & $65.6 \pm 11.7$ & $65.3 \pm 8.6$ & 0.420 \\
\hline Gender & & & & 0.335 \\
\hline $\operatorname{Men}(n, \%)$ & $80(52.3 \%)$ & $76(47.2 \%)$ & $87(55.4 \%)$ & \\
\hline Women $(\mathrm{n}, \%)$ & $73(47.7 \%)$ & $85(52.8 \%)$ & $70(44.6 \%)$ & \\
\hline \multicolumn{5}{|c|}{ Cardiovascular risk factors } \\
\hline $\mathrm{CHD}(\mathrm{n}, \%)$ & $73(47.7 \%)$ & $81(50.3 \%)$ & $82(52.2 \%)$ & 0.727 \\
\hline Hypertension $(\mathrm{n}, \%)$ & $121(79.1 \%)$ & $119(73.9 \%)$ & $117(74.5 \%)$ & 0.509 \\
\hline Diabetes (n, \%) & $66(43.1 \%)$ & $60(37.3 \%)$ & $60(38.2 \%)$ & 0.524 \\
\hline Lipid disorders (n,\%) & $98(64.1 \%)$ & $101(62.7 \%)$ & $108(68.8 \%)$ & 0.494 \\
\hline Smoking (n, \%) & $54(35.3 \%)$ & $64(39.8 \%)$ & $64(40.8 \%)$ & 0.575 \\
\hline \multicolumn{5}{|l|}{ Drug therapy } \\
\hline Antiplatelet drugs (n, \%) & $106(69.3 \%)$ & $101(62.7 \%)$ & $112(71.3 \%)$ & 0.230 \\
\hline Statins (n, \%) & $76(49.7 \%)$ & $88(54.7 \%)$ & $87(55.4 \%)$ & 0.546 \\
\hline $\mathrm{ACEI} / \mathrm{ARB}(\mathrm{n}, \%)$ & $78(51.0 \%)$ & $83(51.6 \%)$ & $82(52.2 \%)$ & 0.976 \\
\hline CCB $(n, \%)$ & $65(42.5 \%)$ & $51(31.7 \%)$ & $54(34.4 \%)$ & 0.118 \\
\hline$\beta$-blocker (n, \%) & $61(39.9 \%)$ & $68(42.2 \%)$ & $50(31.8 \%)$ & 0.137 \\
\hline Diuretics (n, \%) & $46(30.1 \%)$ & $53(32.9 \%)$ & $51(32.5 \%)$ & 0.844 \\
\hline NIHSS Score (mean $\pm S D$ ) & $5.9 \pm 5.2$ & $5.6 \pm 5.0$ & $5.1 \pm 5.3$ & 0.109 \\
\hline \multicolumn{5}{|l|}{ Socioeconomic status } \\
\hline Educational level (n, \%) & & & & $<0.001$ \\
\hline Low & $17(11.1 \%)$ & 0 & 0 & \\
\hline Medium-Low & $47(30.7 \%)$ & $7(4.3 \%)$ & $3(1.9 \%)$ & \\
\hline Medium & $42(27.5 \%)$ & $20(12.4 \%)$ & $10(6.4 \%)$ & \\
\hline Medium-High & $40(26.1 \%)$ & $73(45.3 \%)$ & $58(36.9 \%)$ & \\
\hline High & $7(4.6 \%)$ & $61(37.9 \%)$ & $86(54.8 \%)$ & \\
\hline $\begin{array}{l}\text { Annual income level (n, } \\
\%)\end{array}$ & & & & $<0.001$ \\
\hline Low & $21(13.7 \%)$ & $3(1.9)$ & 0 & \\
\hline Medium-Low & $73(47.7 \%)$ & $47(29.2 \%)$ & $11(7.0 \%)$ & \\
\hline Medium & $53(34.6 \%)$ & $95(59.0 \%)$ & $53(33.8 \%)$ & \\
\hline Medium-High & $6(3.9 \%)$ & $16(9.9 \%)$ & $64(40.8 \%)$ & \\
\hline High & 0 & 0 & $29(18.5 \%)$ & \\
\hline Occupation level (n, \%) & & & & $<0.001$ \\
\hline Low & $16(10.5 \%)$ & $12(7.5 \%)$ & $2(1.3 \%)$ & \\
\hline Medium-Low & $61(39.9 \%)$ & $33(20.5 \%)$ & $10(6.4 \%)$ & \\
\hline Medium & $56(36.3 \%)$ & $80(49.7 \%)$ & $44(28.0 \%)$ & \\
\hline Medium-High & $20(13.1 \%)$ & $35(21.7 \%)$ & $53(33.8 \%)$ & \\
\hline High & 0 & $1(0.6 \%)$ & $48(30.6 \%)$ & \\
\hline $\begin{array}{l}\text { Medical insurance level } \\
(\mathbf{n}, \%)\end{array}$ & & & & $<0.001$ \\
\hline Low & $32(20.9 \%)$ & $10(6.2 \%)$ & $3(1.9 \%)$ & \\
\hline Medium-Low & $58(37.9 \%)$ & $58(36.0 \%)$ & $23(14.6 \%)$ & \\
\hline Medium & $48(31.4 \%)$ & $70(43.5 \%)$ & $58(36.9 \%)$ & \\
\hline Medium-High & $14(9.2 \%)$ & $22(13.7 \%)$ & $60(38.2 \%)$ & \\
\hline High & $1(0.7 \%)$ & $1(0.6 \%)$ & $13(8.3 \%)$ & \\
\hline
\end{tabular}

Approximately one-third (31.0\%) of the patients were with a less than high school educational level, and $75.6 \%$ reported an annual income less than $¥ 60,000$. Among the study population, $38.2 \%$ were retired patients and $28.5 \%$ were peasants, unemployed and manual workers. Most patients $(76.4 \%)$ had a less than 50\% reimbursement percentage. Age, gender, and drug therapy in three 
groups showed no significant difference. The cardiovascular risk factors such as hypertension, diabetes, lipid disorders, and smoking habit were also not significantly different across three tertiles. Although no significant difference was detected among the three tertiles, the stroke severity (NIHSS score) in low SES patients seemed to be higher than in the other groups $(\mathrm{P}=0.109)$.

Table 2. The neighborhood status of the enrolled patients according to the tertiles of individual socioeconomic status

\begin{tabular}{|c|c|c|c|c|}
\hline & \multicolumn{3}{|c|}{ Individual SES tertiles } & \multirow[t]{2}{*}{ P value } \\
\hline & Low $(n=153)$ & Middle (n=161) & High $(n=157)$ & \\
\hline \multicolumn{4}{|c|}{ Neighborhood status } & $<0.001$ \\
\hline \multirow[t]{2}{*}{ Low $(n=137)$} & \#*89 (58.2\%) & $\# \Delta 25(15.5 \%)$ & ${ }^{*} \Delta 23(14.6 \%)$ & $\begin{array}{l}\#<0.001 \\
*<0.001\end{array}$ \\
\hline & & & & $\Delta 0.612$ \\
\hline \multirow[t]{2}{*}{ Middle (n=191) } & \#*43 (28.1\%) & $\# \Delta 78(48.4 \%)$ & ${ }^{*} \Delta 70(44.6 \%)$ & $\begin{array}{l}\#<0.001 \\
* 0.001\end{array}$ \\
\hline & & & & $\Delta 0.109$ \\
\hline \multirow[t]{2}{*}{ High $(n=143)$} & \#²1 (13.7\%) & $\# \Delta 58(36.0 \%)$ & ${ }^{*} \Delta 64(40.8 \%)$ & $\begin{array}{l}\#<0.001 \\
*<0.001\end{array}$ \\
\hline & & & & $\Delta 0.078$ \\
\hline
\end{tabular}

SES, socioeconomic status

\# Low SES tertile vs. middle SES tertile; * low SES tertile vs. high SES tertile; $\triangle$ middle SES tertile vs. high SES tertile

\section{The relationship between socioeconomic status and neighborhood status}

Several significant differences in the neighborhood status were detected across SES tertiles of the participants. There were 137, 191, and 143 patients with low, middle and high neighborhood status, respectively $(\mathrm{P}<0.001$; Table 2$)$. Among low neighborhood status patients, there are significantly more patients with low individual SES (89; 58.2\%) compared with middle $(25 ; 15.5 \%)$ and high (23; $14.6 \%)$ SES tertiles ( $\mathrm{P}<0.001$ vs. middle SES tertile and high SES tertile, respectively; Table 2). In both middle and high neighborhood status groups, patients with low SES were significantly fewer than patients with middle and high SES (Middle neighborhood status group: $\mathrm{P}<0.001$ vs. middle SES tertile and $P=0.001$ vs. high SES tertile, respectively; High neighborhood status group: $\mathrm{P}<0.001$ vs. middle SES tertile and high SES tertile, respectively; Table 2). The proportion of patients with low SES (58.2\%) was significantly higher than that in middle $(28.1 \%)$ and high $(13.7 \%)$ neighborhood status groups (Table 2). We also conducted a correlation analysis between individual SES and neighborhood status. The individual SES showed a significant positive correlation with neighborhood status among the enrolled patients $(r=0.370, \mathrm{P}<0.001)$.

\section{Clinical adverse event rate and all-cause mortality across the tertiles of individual socioeconomic status}

The median follow-up time was 31.6 \pm 10.4 months. 12 patients were lost to follow-up during this period: 5 patients in the low tertile, 3 in the middle tertile, and 4 in the high tertile. 39 patients were died during the follow-ups: 1 low SES patient and 2 high SES patients died due to other causes. The cumulative incidence of clinical adverse events was summarized in Table 3. The incidence of clinical adverse events was higher in low SES patients (60; 39.2\%) when compared with the other patients: middle SES tertile (47; 29.1\%) and high SES tertile (32; 20.3\%). Similarly, patients with a lower individual SES had higher mortality, with survival estimates of $86.9 \%$, 93.2\%, and $94.9 \%$ in increasing tertiles of SES. Inter-group analysis also showed a marked higher incidence of clinical adverse events in low SES patients when compare with two other tertiles $(\mathrm{P}<0.001$ vs. middle tertile and high tertile, respectively; Table 3 ). The inter-group analysis also showed a significantly higher clinical adverse event rate in middle SES patients than that of high SES patients $(P=0.026$, Table 3) Moreover, the all-cause mortality in low SES patients was significantly higher than in middle and high SES tertiles according to the inter-group analysis results $(\mathrm{P}=0.001$ vs. middle tertile and $\mathrm{P}<0.001$ vs. high tertile, respectively; Table 3 ).

Table 3. The incidence of clinical adverse event of the study population.

\begin{tabular}{|c|c|c|c|c|}
\hline \multirow{2}{*}{$\begin{array}{l}\text { Clinical adverse } \\
\text { events }\end{array}$} & \multicolumn{3}{|c|}{ Individual socioeconomic status tertiles } & \multirow[t]{2}{*}{ P value } \\
\hline & Low $(n=153)$ & Middle $(n=161)$ & High $(n=157)$ & \\
\hline Total (n, \%) & $\#^{*} 60(39.2 \%)$ & $\# \Delta 47(29.1 \%)$ & ${ }^{*} \Delta 32(20.3 \%)$ & $\begin{array}{l}\#<0.001 \\
*<0.001 \\
\Delta 0.026\end{array}$ \\
\hline Death (n, \%) & \#²0 (13.1\%) & $\# \Delta 11(6.8 \%)$ & ${ }^{*} \Delta 8(5.1 \%)$ & $\begin{array}{l}\# 0.001 \\
*<0.001 \\
\Delta 0.103\end{array}$ \\
\hline $\begin{array}{l}\text { Nonfatal } \\
\text { recurrence (n, \%) }\end{array}$ & $16(10.5 \%)$ & $14(8.8 \%)$ & $11(7.0 \%)$ & 0.560 \\
\hline $\begin{array}{l}\text { Post-stroke } \\
\text { disability (n, \%) }\end{array}$ & $14(9.2 \%)$ & $13(8.1 \%)$ & $9(5.8 \%)$ & 0.510 \\
\hline $\begin{array}{l}\text { Nonfatal } \\
\text { recurrence + } \\
\text { Post-stroke } \\
\text { disability }(n, \%)\end{array}$ & $10(6.5 \%)$ & $9(5.6 \%)$ & $4(2.5 \%)$ & 0.233 \\
\hline
\end{tabular}

A Kaplan-Meier survival analysis for clinical adverse outcomes showed a significant lower event-free survival rate in patients with a low SES after adjusted age, gender and cardiovascular risks $(\mathrm{P}$ $=0.009$, Figure 2A), and this result still remained statistically significant after furtherly adjusted for education, income, occupation, medical insurance 
reimbursement and neighborhood status $(P=0.017$, Figure 2B). Similarly, a Kaplan-Meier survival analysis for all-cause mortality showed a lower survival rate in low SES patients after adjusted for age, gender and cardiovascular risks $(\mathrm{P}=0.038$, Figure $3 \mathrm{~A})$. This association persisted after adjusted for other factors including education, income, occupation, medical insurance reimbursement and neighborhood status ( $\mathrm{P}=0.040$, Figure 3B).

\section{Multivariate hazards ratio based on individual socioeconomic status and neighborhood status}

The multivariate Cox regression analysis to examine combined influences of individual SES and neighborhood status on clinical outcomes of ischemic stroke patients were shown in Table 4 and Figure 4. Both individual SES (HR 0.767, 95\% CI 0.623-0.944; P = 0.012 ) and neighborhood status (HR 0.730, 95\% CI $0.582-0.916 ; \mathrm{P}=0.007$ ) are independently associated with the clinical outcomes in ischemic stroke patients.
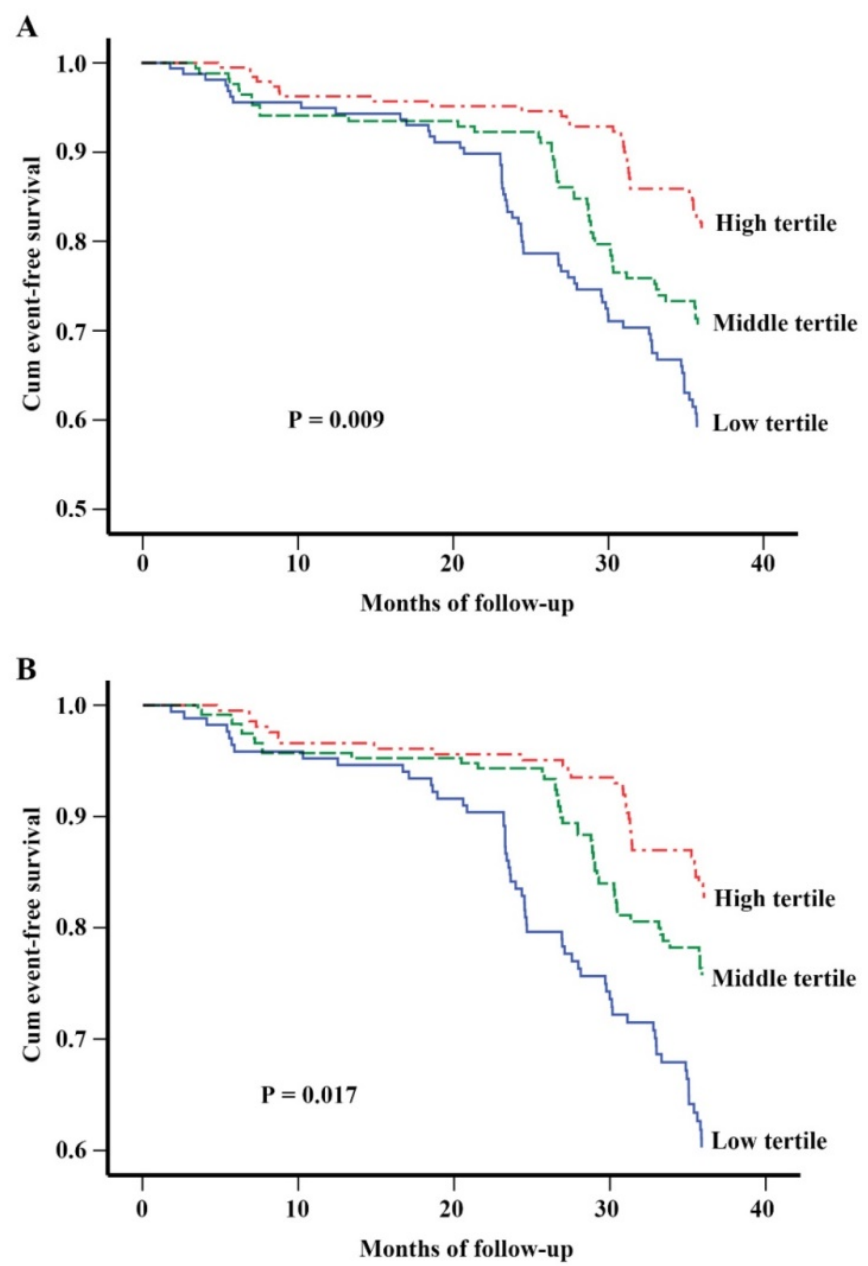

Figure 2 - Multivariable adjusted survival curves for clinical adverse events according to individual SES tertiles. (A) Adjusted for age, gender and cardiovascular risk factors. (B) Adjusted for age, gender, cardiovascular risks, education, income, occupation, medical insurance reimbursement and neighborhood status. Cardiovascular risk factors include CHD, hypertension, diabetes mellitus, lipid disorders and smoking. SES, socioeconomic status; CHD, coronary heart disease.
The HRs of clinical adverse events and all-cause mortality according to different individual SES tertiles and neighborhood status groups were outlined in Table 5. Relative to the high SES tertile, HRs of clinical adverse events and all-cause mortality in low SES patients were significantly high, with a gradually significant increasing HR was observed from high to low tertile in personal SES after adjusted for age, gender and cardiovascular risk factors (Model 1). These results were similar when we conducted the analysis after adjusted for Model 1 plus individual SES and neighborhood status (Model 2). We also detected the relative higher HRs of clinical adverse events and all-cause mortality in patients with low neighborhood status as compared with high neighborhood status when multivariate Cox regression was conducted using both Model 1 and Model 2.
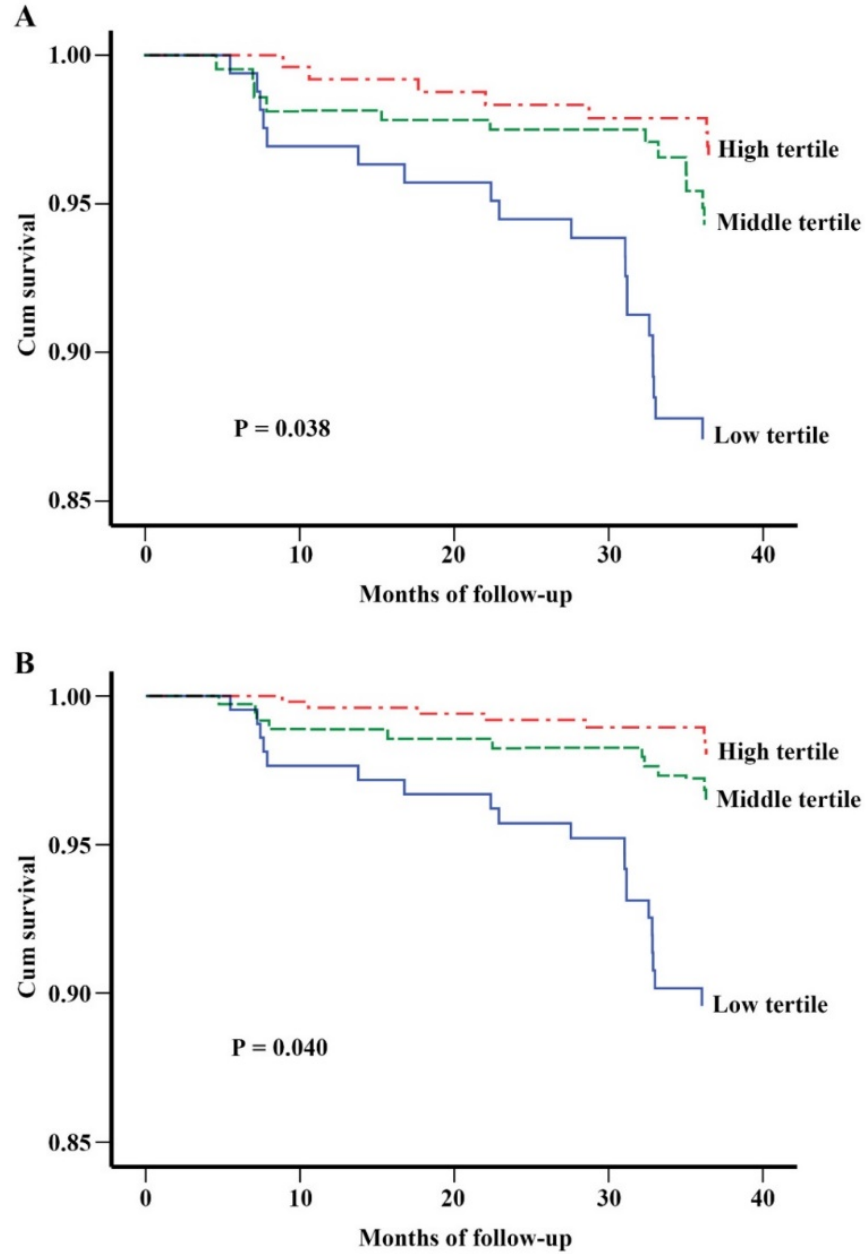

Figure 3 - Multivariable adjusted survival curves for all-cause mortality according to individual SES tertiles. (A) Adjusted for age, gender and cardiovascular risk factors. (B) Adjusted for age, gender, cardiovascular risks, education, income, occupation, medical insurance reimbursement and neighborhood status. Cardiovascular risk factors include CHD, hypertension, diabetes mellitus, lipid disorders and smoking. SES, socioeconomic status; CHD, coronary heart disease. 
Table 4. Adjusted HRs for combined influences of individual and neighborhood SES on clinical outcomes of ischemic stroke patients

\begin{tabular}{|c|c|c|c|c|}
\hline & \multicolumn{2}{|c|}{ Clinical adverse events } & \multicolumn{2}{|c|}{ Mortality } \\
\hline & HR $(95 \%$ CI) & Pvalue & HR $(95 \% \mathrm{CI})$ & Pvalue \\
\hline Age & $0.988(0.973-1.004)$ & 0.132 & $0.976(0.959-0.996)$ & 0.019 \\
\hline Gender & $0.203(0.882-1.640)$ & 0.242 & $1.228(0.825-1.828)$ & 0.311 \\
\hline CHD & $1.023(0.751-1.394)$ & 0.887 & $1.101(0.740-1.638)$ & 0.636 \\
\hline Hypertension & $0.873(0.598-1.274)$ & 0.481 & $0.718(0.427-1.207)$ & 0.212 \\
\hline Diabetes mellitus & $0.821(0.596-1.131)$ & 0.228 & $0.736(0.489-1.107)$ & 0.141 \\
\hline Lipid disorders & $1.041(0.753-1.439)$ & 0.809 & $0.960(0.632-1.459)$ & 0.849 \\
\hline Smoking & $1.052(0.759-1.458)$ & 0.762 & $1.073(0.704-1.635)$ & 0.743 \\
\hline Individual SES & $0.767(0.623-0.944)$ & 0.012 & $0.677(0.515-0.890)$ & 0.005 \\
\hline Neighborhood status & $0.730(0.582-0.916)$ & 0.007 & $0.557(0.410-0.757)$ & $<0.001$ \\
\hline
\end{tabular}

Values are presented as HRs (95\% CI). HRs and 95\% CIs were estimated with multivariate Cox regression analysis.

$\mathrm{HR}$, hazard ratio; CHD, coronary heart disease; SES, socioeconomic status; CI, confidence interval.

Table 5. Adjusted HRs for clinical outcomes in ischemic stroke patients according to individual SES and neighborhood status

\begin{tabular}{|c|c|c|c|c|c|c|c|c|}
\hline \multirow[t]{3}{*}{ Variables } & \multicolumn{4}{|c|}{ Clinical adverse events } & \multicolumn{4}{|c|}{ All-cause mortality } \\
\hline & Model 1* & & Model 2\# & & Model 1* & & Model 2\# & \\
\hline & $\mathrm{HR}(95 \% \mathrm{CI})$ & $P$ value & HR $(95 \% \mathrm{CI})$ & $P$ value & HR (95\% CI) & $P$ value & HR $(95 \% \mathrm{CI})$ & $P$ value \\
\hline \multicolumn{9}{|c|}{ Individual SES } \\
\hline Low & $2.127(1.427-3.171)$ & $<0.001$ & $1.736(1.129-2.668)$ & 0.012 & $3.057(1.817-5.143)$ & $<0.001$ & $2.127(1.220-3.710)$ & 0.008 \\
\hline Middle & $1.512(1.003-2.281)$ & 0.048 & $1.495(0.991-2.255)$ & 0.056 & $1.421(0.801-2.520)$ & 0.229 & $1.414(0.797-2.509)$ & 0.236 \\
\hline High & Reference & & Reference & & Reference & & Reference & \\
\hline \multicolumn{9}{|c|}{ Neighborhood status } \\
\hline Low & $2.326(1.504-3.596)$ & $<0.001$ & $1.954(1.222-3.126)$ & 0.005 & $4.085(2.236-7.363)$ & $<0.001$ & $3.053(1.619-5.760)$ & 0.001 \\
\hline Middle & $1.500(0.978-2.302)$ & 0.063 & $1.450(0.946-2.223)$ & 0.089 & $1.645(0.886-3.054)$ & 0.115 & $1.567(0.845-2.908)$ & 0.154 \\
\hline High & Reference & & Reference & & Reference & & Reference & \\
\hline
\end{tabular}

Values are presented as HRs (95\% CI). HRs and 95\% CIs were estimated with multivariate Cox regression analysis.

HR, hazard ratio; SES, socioeconomic status; CI, confidence interval.

*Adjusted for age, gender, cardiovascular risks. \#Adjusted for age, gender, cardiovascular risks, individual SES, neighborhood SES. Cardiovascular risk factors include CHD, hypertension, diabetes mellitus, lipid disorders and smoking.

Table 6. Adjusted HRs for clinical outcomes according to neighborhood status among individual SES in ischemic stroke patients

\begin{tabular}{|c|c|c|c|c|c|}
\hline \multirow[t]{2}{*}{ Individual SES } & \multirow[t]{2}{*}{ Neighborhood status } & \multicolumn{2}{|c|}{ Clinical adverse events } & \multicolumn{2}{|l|}{ All-cause mortality } \\
\hline & & HR (95\% CI) & P value & HR $(95 \% \mathrm{CI})$ & P value \\
\hline \multirow[t]{3}{*}{ Low } & Low & $1.550(0.593-4.051)$ & 0.372 & $3.570(0.925-13.782)$ & 0.065 \\
\hline & Middle & $1.506(0.605-3.749)$ & 0.379 & $2.339(0.606-9.031)$ & 0.218 \\
\hline & High & Reference & & Reference & \\
\hline \multirow[t]{3}{*}{ Middle } & Low & $2.237(0.775-6.451)$ & 0.137 & $1.818(0.394-8.403)$ & 0.444 \\
\hline & Middle & $1.786(0.560-5.682)$ & 0.327 & $1.091(0.215-5.541)$ & 0.917 \\
\hline & High & Reference & & Reference & \\
\hline \multirow[t]{3}{*}{ High } & Low & $4.477(0.858-23.366)$ & 0.075 & $5.280(0.539-51.734)$ & 0.153 \\
\hline & Middle & $1.987(0.564-6.995)$ & 0.285 & $1.298(0.203-8.301)$ & 0.783 \\
\hline & High & Reference & & Reference & \\
\hline
\end{tabular}

Values are presented as HRs (95\% CI). HRs and 95\% CIs were estimated with multivariate Cox regression analysis adjusted for age, gender, cardiovascular risk factors, education, household income, occupation, medical insurance reimbursement and neighborhood status.

HR, hazard ratio; SES, socioeconomic status; CI, confidence interval.

The effects of neighborhood status on clinical outcomes in ischemic stroke patients based on different tertiles of individual SES are shown in Table 6 . However, the multivariate Cox regression showed no significant difference in HRs of both clinical adverse events and all-cause mortality according to neighborhood status by individual SES after adjusted for age, gender, cardiovascular risk factors, education, household income, occupation, medical insurance reimbursement and neighborhood status. The effects of individual SES on clinical outcomes in ischemic stroke patients with exclusion of influences of the neighborhood status was shown in Table 7. The HRs of clinical adverse events exhibited a significant increase in patients with lower individual SES in the low neighborhood status group (low tertile: HR 1.912, 95\% CI 1.100-3.322; P $=0.022$ and middle tertile: HR $1.031,95 \%$ CI 1.012-1.075; $\mathrm{P}=0.044)$. There also existed a significantly increased HRs of all-cause mortality in lower SES patients with low neighborhood status (low tertile: HR 2.074, 95\% CI 1.103-3.906; $\mathrm{P}=0.024$ and middle tertile: $\mathrm{HR} 1.038$, 
95\% CI 1.003-1.075; P = 0.033). A similar higher HR of clinical adverse events and all-cause mortality was found in low SES patients with middle neighborhood status $(\mathrm{P}=0.026$ and $\mathrm{P}=0.039$, respectively). Although the other results did not reach the statistical significance, the multivariate Cox regression analysis results tended to show lower individual SES as well as poorer neighborhood status being associated with an increase in clinical adverse events and all-cause mortality HRs in ischemic stroke patients. Combined with the data in Table 6 and 7, the findings simultaneously suggested that individual SES may be a more important risk factor than neighborhood status in ischemic stroke.

\section{Discussion}

The present study examined whether individual SES was associated with neighborhood status and explored the influences of SES on the clinical outcomes in ischemic stroke patients. The main findings of this retrospective study can be summarized as follows: (1) the individual SES was significantly correlated with neighborhood status in patients with ischemic stroke; (2) both individual SES and neighborhood status of the patients are the important independent predictors of clinical adverse events and all-cause mortality in ischemic stroke; and (3) low SES patients with a poorer neighborhood status tended to present worse clinical outcomes compared with the other patients in the long-term follow-up.

China has the largest patient population of stroke patients in the world and ischemic stroke is regarded as a major cause of morbidity and mortality as well as a substantial health care burden with increase of aging population and changes of lifestyle in last decades [26]. All factors influence the clinical outcomes and mortality should be taken into consideration to improve the stroke care.
Socioeconomic-related inequalities in healthcare could also lead to disparities in management of ischemic stroke patients, since components of SES could play important roles in psychology, behavior and physical functions [27, 28]. Previous studies have observed the association of SES with mortality in ischemic stroke patients. In Canada, a study reported a low income could cause an increase in the mortality in ischemic stroke during one-year follow-up when compared with higher income groups [29]. Qureshi et al. [30] have found that educational level is an independent factor in clinical outcomes and has a significant effect on the risk for stroke. SES could also potentially change patients' behavioral manners and lifestyles such as following doctors' advices and exercises for recovery that were related with healthcare [31]. Lower SES patients may have poor awareness of risk factors for diseases due to lack of education and health knowledge and therefore lead to worse outcomes [32]. Other studies also indicated that unemployed could potentially increase the short-term mortality in ischemic stroke and occupational status are interrelated with household income and educational level that could exert a synergistic effect $[9,12]$. However, most of these studies were conducted in the Western countries, as some indicators for SES may not applicable in China, such as educational level, which can be a proxy for personal SES in many developed countries, was not suitable in China [33, 34]. In China, there were relatively fewer studies that detailed the relationship between SES and the clinical outcomes after ischemic stroke. In the Nanjing Stroke Registry Program study, the results showed a lower survival rate after first-ever ischemic stroke was closely associated with low levels of household income, occupational class, and housing space, but not with educational levels [35].

Table 7. Adjusted HRs for clinical outcomes according to individual SES among neighborhood status in ischemic stroke patients

\begin{tabular}{|c|c|c|c|c|c|}
\hline \multirow{2}{*}{ Neighborhood status } & \multirow{2}{*}{ Individual SES } & \multicolumn{2}{|l|}{ Clinical adverse events } & \multicolumn{2}{|l|}{ All-cause mortality } \\
\hline & & HR (95\% CI) & P value & HR $(95 \% \mathrm{CI})$ & P value \\
\hline \multirow[t]{3}{*}{ Low } & Low & $1.912(1.100-3.322)$ & 0.022 & $2.074(1.103-3.906)$ & 0.024 \\
\hline & Middle & $1.031(1.012-1.075)$ & 0.044 & 1.038 (1.003-1.075) & 0.033 \\
\hline & High & Reference & & Reference & \\
\hline \multirow[t]{3}{*}{ Middle } & Low & $2.096(1.092-4.026)$ & 0.026 & $2.628(1.050-6.581)$ & 0.039 \\
\hline & Middle & $1.315(0.718-2.409)$ & 0.376 & $1.609(0.668-3.873)$ & 0.289 \\
\hline & High & Reference & & Reference & \\
\hline \multirow[t]{3}{*}{ High } & Low & $1.983(0.716-5.492)$ & 0.188 & $1.664(0.399-6.939)$ & 0.485 \\
\hline & Middle & $1.457(0.643-3.304)$ & 0.367 & $1.024(0.315-3.289)$ & 0.969 \\
\hline & High & Reference & & Reference & \\
\hline
\end{tabular}

Values are presented as HRs (95\% CI). HRs and 95\% CIs were estimated with multivariate Cox regression analysis adjusted for age, gender, cardiovascular risk factors, and individual SES.

$\mathrm{HR}$, hazard ratio; SES, socioeconomic status; $\mathrm{CI}$, confidence interval. 
A

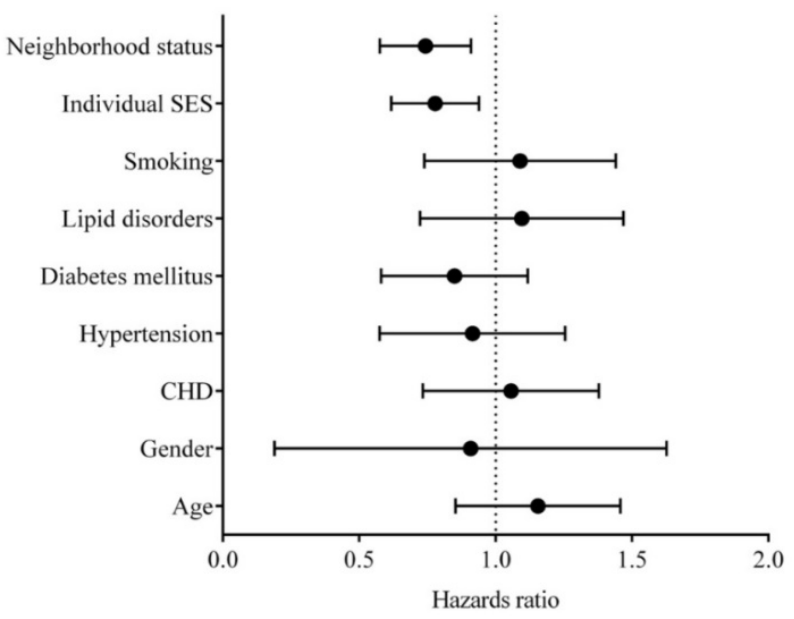

B

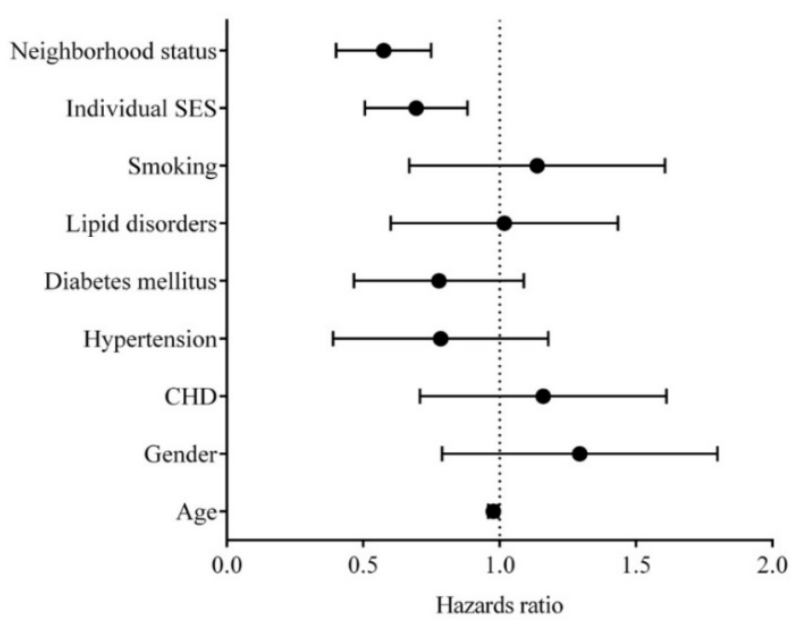

Figure 4 - Adjusted HRs for clinical adverse events and all-cause mortality of ischemic stroke patients. (A) Multivariate Cox regression analysis demonstrated both individual SES and neighborhood status are independent correlated of clinical adverse events. (B) Multivariate Cox regression analysis revealed that age, individual SES and neighborhood status are all important predictors for all-cause mortality in ischemic stroke. HR, hazard ratio; SES, socioeconomic status; CHD, coronary heart disease.

Evidence from the nationally representative China National Stroke Registry (CNSR) study demonstrated significant inequalities in survival after stroke due to individual and combined distinctions in education level, occupational class, and household income and patients with high SES tended to have better outcomes [36]. Our data indicated similar results to CNSR by using a combined SES score which has also taken into account of health insurance as an important component for SES, since our previous study showed that health insurance was an important prognostic factor in cardiovascular diseases, especially in rural areas [17].

In our present study, we observed a close association between individual SES and neighborhood status. In Shanghai, China, patients with low neighborhood status are now generally divided into two different populations according to their hukou registered locations. The first population refers to patients with a Shanghai hukou and from rural areas; the second population refers to the rural migrant workers, or "the third population cohort", who have moved from other cities to grasp new occupational, educational and medical opportunities in the past few decades but did not carry a Shanghai registered hukou location [37]. Although these migrant workers may not live in the rural areas of Shanghai, their healthcare still mainly depend on the related policies issued in their original rural hukou locations and thus were also regarded as having a lower neighborhood status compared to urban residents because of the low incomes, educational and occupational levels $[16,20]$. According to our data, approximately $60 \%$ low SES patients were with low neighborhood status and less than 15\% high SES patients were classified as low neighborhood. Compared with higher neighborhood status, a poorer neighborhood status could also predict a worse clinical outcome as well as all-cause mortality. This apparent difference in the prognosis of ischemic stroke was actually a reflection of the now-existing urban-rural health inequality. Despite the current China's healthcare reform have put a lot of efforts into reducing costs and improving patient assistance, urban-rural health inequality is still a problem with great political importance that cannot be ignored. According to the new nationwide longitudinal survey data household wealth in China, the mean annual household income per person in a rural/urban family was $¥ 7,917 / ¥ 24,565$ in year 2012 [38]. However, the out-of-pocket cost for an average hospitalization is similar to the China's per capita annual income [39]. Moreover, both the health insurance coverage and reimbursement percentage were relatively lower in the rural areas [17]. Thus, several rural patients cannot afford such a great burden and choose to discharge early from the hospital. In addition, stroke-targeted necessary drugs after discharge is also a tremendous medical cost in the long run and patients from rural areas were less adherent to scheduled stroke medications [17]. Taking into together, these financial barriers may limit effective therapies and result in poorer clinical outcomes. Besides, a life-threatening disease accompanied with expensive medical cost could cause mental illnesses such as anxiety and depression among the low SES group and affect the therapy [27]. Another reason for the clinical worsening among the patients with low neighborhood status is the delay in accessing timely and effective treatment after stroke. Although at least a community health-care center in each of the rural areas of China was established to provide preliminary 
healthcare services, these community hospitals still lack expertise and technology to care for stroke patients, since stroke has been generally regarded as a critical illness that is best diagnosed and treated in senior hospitals. This inconvenience in achieving therapeutic measures could lead to the unwillingness of lower neighborhood status patients to comply with treatment and cooperate with their doctors and thus have a great effect on stroke care.

The findings in our study provide not only a new insight to reconsider the risk factors for ischemic stroke, but also a suggestion for China's healthcare reform in the future. Physicians should have perceptions of potential risk factors and severity associated with low SES as well as low neighborhood status and choose effective therapeutic methods to improve secondary prevention and stroke care among these patients. In the meantime, related policies should focus on alleviation of socioeconomic medical burden and improvement of healthcare services through increasing health insurance coverage and reimbursement, reducing medical cost and providing medical allowances among low SES patients which could lessen the health inequalities aforementioned.

Although we have examined the roles of individual SES in ischemic stroke patients with different neighborhood status, the mechanisms through which SES affects clinical outcomes are believed to extend further. Besides, our findings indicated individual SES may be a more important risk factor than neighborhood status in clinical outcomes of ischemic stroke. It is possibly because individual SES is a relatively broader notion covering a wide range of aspects that closely associated with healthcare than neighborhood status. Moreover, individual SES could also partially mediate the associations between neighborhood status and health outcomes [19]. Although the risk of neighborhood status was reduced in the regression model based on different SES tertiles, the neighborhood status was also significantly correlated with SES and was an independent risk factor for ischemic stroke in a pooled multivariate Cox regression analysis. Since economic burden, inequitable distribution of healthcare services, and other factors are global medical problems that also existed in developed countries, further studies should be conducted to elucidate the relationship between SES and ischemic stroke.

Several limitations of our study warrant discussion. Firstly, this is a retrospective study from a single center in a tertiary hospital. The sample size was small and the follow-up period was relatively short. Secondly, we did not analyze the levels of education, occupation, and health insurance according to different neighborhood status. However, our data have demonstrated neighborhood status was significantly correlated with personal SES and could be an independent risk factor, which could also explain the roles of neighborhood status in ischemic stroke. Thirdly, a few patients are temporary residents who are old parents living with their married sons/daughters to look after grandchildren, while the sons/daughters take care of their parents' healthy conditions. Selection of these patients may possibly cause bias to our study results, but it seems minimal since we classified the neighborhood status according to their hukou registered locations which hugely influences their healthcare-related socioeconomic gradients. Despite the limitations of our approach, it is likely that individual SES could be also used as an important prognostic factor in ischemic stroke patients with different neighborhood status.

\section{Conclusion}

In summary, we have found that individual SES was significantly associated with neighborhood status among ischemic stroke patients. Both individual SES and neighborhood status are independently associated with ischemic stroke and patients with a lower SES as well as poorer neighborhood status may have a significantly increased risk for adverse clinical outcomes. Continuous healthcare reform should properly consider the potential influences of lower SES and tackle health inequality to warrant a better therapy in ischemic stroke patients.

\section{Abbreviations}

SES: socioeconomic status; CT: computed tomography; MRI: magnetic resonance imaging; CHD: coronary heart disease; HDL: high density lipoprotein; LDL: low density lipoprotein; SD: standard deviation; HR: hazards ratio; CI: confidence interval; CNSR: China National Stroke Registry.

\section{Acknowledgements}

This study was supported by the National Natural Science Foundation of China (no. 81371212 to Xueyuan Liu) and Shanghai Science and Technology Committee Research Projects, China (no. 13411951102 and no. 13JC1404002 to Xueyuan Liu). Both Han Yan and Baoxin Liu were supported by International Exchange Program for Graduate Student, Tongji University, China (no. 2015020041 and no. 2015020040). The author would like to thank all participants in this study for their active cooperation.

\section{Competing Interests}

The authors have declared that no competing interest exists. 


\section{References}

1. Wu Z, Yao C, Zhao D, et al. Sino-MONICA Project: a collaborative study on trends and determinants in cardiovascular diseases in China, Part I: morbidity and mortality monitoring. Circulation. 2001; 103: 462-8.

2. Tsai CF, Thomas B, Sudlow CL. Epidemiology of stroke and its subtypes in Chinese vs white populations: a systematic review. Neurology. 2013; 81: 264-72.

3. Wolfe CD, Rudd AG, Howard R, et al. Incidence and case fatality rates of stroke subtypes in a multiethnic population: the South London Stroke Register. J Neurol Neurosurg Psychiatry. 2002; 72: 211-6.

4. Jakovljevic D, Sarti C, Sivenius J, et al. Socioeconomic status and ischemic stroke: the FINMONICA Stroke Register. Stroke. 2001; 32: 1492-8.

5. Clark AM, DesMeules M, Luo W, et al. Socioeconomic status and cardiovascular disease: risks and implications for care. Nat Rev Cardiol. 2009; 6: 712-22.

6. Avendano M, Kawachi I, Van Lenthe F, et al. Socioeconomic status and stroke incidence in the US elderly: the role of risk factors in the EPESE study. Stroke. 2006; 37: 1368-73.

7. McCarron P, Greenwood R, Elwood P, et al. The incidence and aetiology of stroke in the Caerphilly and Speedwell Collaborative Studies II: risk factors for ischaemic stroke. Public Health. 2001; 115: 12-20.

8. Kerr GD, Slavin $\mathrm{H}$, Clark $\mathrm{D}$, et al. Do vascular risk factors explain the association between socioeconomic status and stroke incidence: a meta-analysis. Cerebrovasc Dis. 2011; 31: 57-63.

9. Langagergaard V, Palnum $\mathrm{KH}, \mathrm{Mehnert} \mathrm{F}$, et al. Socioeconomic differences in quality of care and clinical outcome after stroke: a nationwide population-based study. Stroke. 2011; 42: 2896-902.

10. van den Bos GA, Smits JP, Westert GP, et al. Socioeconomic variations in the course of stroke: unequal health outcomes, equal care? J Epidemiol Community Health. 2002; 56: 943-8.

11. Sturm JW, Donnan GA, Dewey HM, et al. Determinants of handicap after stroke: the North East Melbourne Stroke Incidence Study (NEMESIS). Stroke. 2004; 35: 715-20.

12. Winkleby MA, Jatulis DE, Frank E, et al. Socioeconomic status and health: how education, income, and occupation contribute to risk factors for cardiovascular disease. Am J Public Health. 1992; 82: 816-20.

13. Wu J, Liu Y, Rao K, et al. Education-related gender differences in health in rural china. Am J Public Health. 2004; 94: 1713-6.

14. Hanibuchi T, Nakaya T, Murata C. Socio-economic status and self-rated health in East Asia: a comparison of China, Japan, South Korea and Taiwan. Eur J Public Health. 2012; 22: 47-52.

15. Tang S, Meng $\mathrm{Q}$, Chen $\mathrm{L}$, et al. Tackling the challenges to health equity in China. Lancet. 2008; 372: 1493-501.

16. Chen R, Wei L, Hu Z, et al. Depression in older people in rural China. Arch Intern Med. 2005; 165: 2019-25.

17. Liu $\mathrm{B}$, Yan $\mathrm{H}$, Guo $\mathrm{R}$, et al. The basic social medical insurance is associated with clinical outcomes in the patients with ST-elevation myocardial infarction: a retrospective study from Shanghai, China. Int J Med Sci. 2014; 11: 905-17.

18. Wang, XR, Hui ECM, Choguill C, et al. The new urbanization policy in China: Which way forward?. Habitat International. 2015; 47: 279-84.

19. Stafford M, Marmot M. Neighbourhood deprivation and health: does it affect us all equally?. Int J Epidemiol. 2003; 32: 357-66.

20. Lo CC, Cheng TC, Bohm M, et al. Rural-to-Urban Migration, Strain, and Juvenile Delinquency: A Study of Eighth-Grade Students in Guangzhou, China. Int J Offender Ther Comp Criminol. 2016; [Epub ahead of print].

21. Kapral MK, Wang H, Mamdani M, et al. Effect of socioeconomic status on treatment and mortality after stroke. Stroke. 2002; 33: 268-73.

22. McKevitt $C$, Coshall $C$, Tilling $K$, et al. Are there inequalities in the provision of stroke care? Analysis of an inner-city stroke register. Stroke. 2005; 36: 315-20.

23. Jauch EC, Saver JL, Adams HP Jr. et al. Guidelines for the early management of patients with acute ischemic stroke: a guideline for healthcare professionals from the American Heart Association/American Stroke Association. Stroke. 2013; 44: 870-947.

24. Sacco RL, Kasner SE, Broderick JP. et al. An updated definition of stroke for the 21st century: a statement for healthcare professionals from the American Heart Association/American Stroke Association. Stroke. 2013; 44: 2064-89.

25. Duncan PW, Lai SM, Keighley J. Defining post-stroke recovery: implications for design and interpretation of drug trials. Neuropharmacology. 2000; 39: 835-41.

26. Liu L, Wang D, Wong KS et al. Stroke and stroke care in China: huge burden, significant workload, and a national priority. Stroke. 2011; 42: 3651-4.

27. Wu WH, Yang L, Peng FH, et al. Lower socioeconomic status is associated with worse outcomes in pulmonary arterial hypertension. Am J Respir Crit Care Med. 2013; 187: 303-10.

28. Adler NE, Boyce T, Chesney MA, et al. Socioeconomic status and health. The challenge of the gradient. Am Psychol 1994; 49: 15-24

29. Kapral MK, Fang J, Chan C, et al. Neighborhood income and stroke care and outcomes. Neurology. 2012; 79: 1200-7.

30. Qureshi AI, Suri MF, Saad M, et al. Educational attainment and risk of stroke and myocardial infarction. Med Sci Monit. 2003; 9: CR466-73.

31. Adler NE, Ostrove JM. Socioeconomic status and health: what we know and what we don't. Ann N Y Acad Sci. 1999; 896: 3-15.
32. Lantz PM, House JS, Lepkowski JM, et al. Socioeconomic factors, health behaviors, and mortality: results from a nationally representative prospective study of US adults. JAMA. 1998; 279: 1703-8.

33. Grube MM, Koennecke HC, Walter G, et al. Association between socioeconomic status and functional impairment 3 months after ischemic stroke: the Berlin Stroke Register. Stroke. 2012; 43: 3325-30.

34. Braveman PA, Cubbin C, Egerter S, et al. Socioeconomic status in health research: one size does not fit all. JAMA. 2005; 294: 2879-88.

35. Zhou G, Liu X, Xu G, et al. The effect of socioeconomic status on three-year mortality after first-ever ischemic stroke in Nanjing, China. BMC Public Health. 2006; 6: 227.

36. Pan $\mathrm{Y}$, Song $\mathrm{T}$, Chen $\mathrm{R}, \mathrm{Li} \mathrm{H}$, et al. Socioeconomic deprivation and mortality in people after ischemic stroke: The China National Stroke Registry. Int J Stroke. 2016; 11: 557-64.

37. Jordan LP, Ren Q, Falkingham J. Youth education and learning in twenty-first century China disentangling the impacts of migration, residence, and hukou. Chin Sociol Rev. 2014; 47: 57-83.

38. Xie Y, Jin Y. Household wealth in China. Chin Sociol Rev. 2015; 47: 203-229.

39. Hu S, Tang S, Liu Y, et al. Reform of how health care is paid for in China: challenges and opportunities. Lancet. 2008; 372: 1846-53 